Research Paper

\title{
Legumain Promotes Gastric Cancer Progression Through Tumor-associated Macrophages In vitro and In vivo
}

\author{
Hongbin Wang1,2, Binghong Chen³, Yingying Lin³, Yi Zhou'1, Xiaobo Li1 ${ }^{\bowtie}$ \\ 1. Division of Gastroenterology and Hepatology, Key Laboratory of Gastroenterology and Hepatology, Ministry of Health, Shanghai Institute of Digestive \\ Disease, Renji Hospital, School of Medicine, Shanghai Jiao Tong University, Shanghai, China. \\ 2. Department of Gastroenterology, Punan Hospital, Pudong New Area, Shanghai, China. \\ 3. Department of Neurosurgery, Renji Hospital, School of Medicine, Shanghai Jiao Tong University, Shanghai, China.
}

$\square$ Corresponding author: Xiaobo Li, M.D. Division of Gastroenterology and Hepatology, Key Laboratory of Gastroenterology and Hepatology, Ministry of Health, Shanghai Institute of Digestive Disease, Renji Hospital, School of Medicine, Shanghai Jiao Tong University, Shanghai, China. Email address: lixiaobo@renji.com

(c) The author(s). This is an open access article distributed under the terms of the Creative Commons Attribution License (https://creativecommons.org/licenses/by/4.0/). See http://ivyspring.com/terms for full terms and conditions.

Received: 2019.05.07; Accepted: 2019.10.14; Published: 2020.01.01

\begin{abstract}
Tumor-associated macrophages (TAMs) play a crucial role in the tumor microenvironment. Legumain (LGMN) has been shown to be a tumor-promoting protein, but the effect of LGMN on TAMs in the progression of gastric cancer (GC) is under exploration. Our studies included the construction of LGMN-knockdown and LGMN-overexpressing TAMs induced from the human cell line THP-1 (PMA/IL-4/IL-13) and murine cell line Raw264.7 (IL-4/IL-13). A CCK-8 assay and transwell migration assay indicated that upregulation of LGMN expression in TAMs stimulated cell proliferation, migration and invasion in vitro, while downregulation of LGMN expression reduced cell proliferation, migration and invasion. In vivo experiments revealed slower growth, less angiogenesis, and less Ki67 expression in LGMN-knockdown TAMs injected with gastric cancer cells compared to control TAMs injected with GC cells. Together, these study results suggested that $\mathrm{LGMN}^{+}$TAMs, which may serve as a potential target for GC treatment, promoted gastric cancer cell proliferation and angiogenesis in vitro and in vivo.
\end{abstract}

Key words: Legumain; gastric cancer; tumor-associated macrophages; angiogenesis

\section{Introduction}

There are pronounced global epidemiological variations in gastric cancer (GC) incidence, with higher incidence rates in East Asia, Eastern Europe and South America than in the rest of the world [1]. Globally, gastric cancer ranked fifth in cancer incidence and second in mortality, and there were 984 000 incident cases of GC and 841000 deaths in 2013 [2]. Population growth and aging resulted in a larger number of cases of stomach cancers in 2013 compared with 1990; although part of this increase was offset by falling rates, GC is still the major cancer worldwide, comprising the global disease burden [2-4]. In areas where GC screening methods (such as endoscopic screening) are not universal, GC is often found at an advanced stage by nonspecific symptoms $[5,6]$, which leads to poor overall survival. Tumor metastasis is a key factor affecting the prognosis of GC; for metastatic patients, systemic chemotherapy with or without biological agents represents the standard of care, contributing to improved overall survival and an improved quality of life $[7,8]$.

Macrophages are one of the most abundant inflammatory cells in the tumor microenvironment [9, 10]. Substantial evidence suggests that macrophages adopt a protumorigenic phenotype rather than a tumoricidal phenotype and function as a vital driver of tumor-promoting inflammation, leading to tumor progression by various methods including promoting 
genetic instability, facilitating metastasis, supporting cancer stem cells and inhibiting protective immunity [11-13]. Legumain (LGMN), also known as asparagine endopeptidase (AEP), is a lysosomal cysteine protease originally identified in the seeds of legumes, which is also present in the human body and is associated with a variety of tumor types at the stages of development, invasion and metastasis [14-16]. Numerous studies have confirmed that LGMN is a potential prognostic factor in GC $[17,18]$. What is the role of LGMN in macrophages in the progression and metastasis of GC? The present study explored the relationship of LGMN expressed by macrophages with GC metastasis in vitro and in vivo.

\section{Materials and Methods}

Cell culture. Human acute monocytic leukemia cells, THP-1 cells (Cat. CBP60518, Cobioer, Nanjing, China), and mouse macrophages, RAW 264.7 cells (Cat. CBP60533, Cobioer, Nanjing, China), were cultured in Dulbecco's modified Eagle's medium (HyClone; GE Healthcare) supplemented with 10\% fetal bovine serum (Gibco; Thermo Fisher Scientific, Inc.) and maintained in a humidified atmosphere at $37^{\circ} \mathrm{C}$ with $5 \% \mathrm{CO}_{2}$. THP-1 cells were activated and differentiated into macrophages by incubation with phorbol-12-myristate-13-acetate (PMA; $100 \mathrm{ng} / \mathrm{ml}$ in complete medium) and IL-4/IL-13 for 3 days, while RAW 264.7 cells were treated with IL-4/IL-13 only. The culture medium was exchanged every day.

Lentiviral vector-mediated gene overexpression or knockdown. An LGMN overexpression sequence was constructed by Hanyin Ltd., Co (Shanghai, China). A recombinant lentivirus and negative control (NC) lentivirus were prepared and titered to $10^{9}$ transfection units $/ \mathrm{ml}$. After $48 \mathrm{~h}$, the efficiency of overexpression was confirmed via RT-qPCR. To obtain stably transfected cells (LGMN-OE), macrophages were seeded in six-well dishes at a density of $1 \times 10^{5}$ cells per well. The cells were then infected with the same virus titer on the following day and treated with $8 \mu \mathrm{g} / \mathrm{ml}$ polybrene. At $72 \mathrm{~h}$ post-viral infection, the culture medium was replaced with a selection medium containing $4 \mu \mathrm{g} / \mathrm{ml}$ puromycin. The puromycin-resistant cells were amplified in a medium containing $2 \mu \mathrm{g} / \mathrm{ml}$ puromycin for 7 days and then transferred to a medium without puromycin. To downregulate the expression of LGMN in both macrophage cell lines, two different LGMN shRNA sequences were cloned into the pTRIPZ plasmid (Open Biosystems, RHS4750, Huntsville, Alabama, USA) according to the manufacturer's instructions. An shRNA sequence targeting LGMN was cloned into the plvx-shRNA plasmid. A non-silencing lentiviral shRNA vector was used as a control. The lentiviruses were packaged using psPAX2 and pMD2G, a three-plasmid system. To obtain stable cell lines, lentivirus supernatant was added to THP-1 and Raw264.7 cells, followed by screening with $1 \mu \mathrm{g} / \mathrm{ml}$ puromycin for 2 weeks. The expression of LGMN was downregulated in these cell lines when the cells were treated for longer than 4 days with $1 \mu \mathrm{g} / \mathrm{ml}$ doxycycline (Dox), an analog of tetracycline, in the culture medium. To overexpress LGMN in THP-1 and Raw264.7 cells, LGMN was cloned into the pLVX-IRES-ZsGeen1 plasmid. Lentivirus supernatant was added to the culture medium of THP-1 and Raw264.7 cells. The infection rate was assessed using a fluorescence microscope.

Western blot analysis. Total protein was extracted from cells with a cell lysis buffer $(50 \mathrm{mM}$ Tris- $\mathrm{HCl} \mathrm{pH}$ 8.0, $120 \mathrm{mM} \mathrm{NaCl}, 0.5 \% \mathrm{NP}-40$, and $1 \mathrm{mM} \mathrm{PMSF}$ ) and evaluated by BCA methods. Protein $(30 \mu \mathrm{g})$ was subjected to $10 \%$ SDS-polyacrylamide gel electrophoresis and then transferred to polyvinylidene difluoride membranes (EMD Millipore, Billerica, MA, USA). The membranes were incubated with a blocking buffer $(5 \%$ skim milk in TBS-T) at room temperature for an hour. After that, the membranes were incubated with the following antibodies at a 1:500 dilution overnight at $4^{\circ} \mathrm{C}$ : an anti-LGMN antibody (cat. no. 67017-1-Ig; ProteinTech Group, Inc., Chicago, IL, USA) and an anti- $\beta$-actin antibody (cat. no. 4970; Cell Signaling Technology, Inc., Beverly, MA, USA). The membranes were washed with TBS-T and then incubated with a horseradish peroxidase-conjugated anti-rabbit or anti-mouse antibody (1:10,000 dilution; Sigma-Aldrich; Merck KGaA, Darmstadt, Germany) at room temperature for $2 \mathrm{~h}$. Detection was performed using western blot detection reagents (Odyssey; LI-COR Biosciences, Lincoln, NE, USA).

Cell proliferation and motility or invasion assays A Cell Counting Kit-8 (CCK-8) assay was performed to assess cell proliferation. Briefly, transfected PMA-treated THP-1 and RAW 264.7 cells were plated at a density of $1 \times 10^{4}$ cells/well in a 96-well plate. Then, $10 \mu \mathrm{L}$ of CCK-8 solution was added to each well and incubated for $2 \mathrm{~h}$. Next, absorbance values were detected at a wavelength of $450 \mathrm{~nm}$ using a Bio-Rad microplate reader. Cell viability was expressed as the optical density (OD) values of the treated groups/OD values of the control groups $\times 100 \%$.

A Transwell migration assay was employed to evaluate cell invasion. 24-well Transwell plates with 8 - $\mu$ m-diameter filters (Coring, NY, USA) were utilized. Approximately $2 \times 10^{5}$ cells suspended in 200 $\mu l$ of serum-free medium were placed in the upper chamber, and $750 \mu 1$ of $10 \%$ FBS medium was added to the lower chamber. The plate was incubated for $8 \mathrm{~h}$ 
at $37^{\circ} \mathrm{C}$ with $5 \% \mathrm{CO}_{2}$. Then, the cells on the upper side were carefully removed with a cotton swab. The cells that passed through the filter were fixed in $40 \mathrm{~g} / \mathrm{L}$ methanol for $15 \mathrm{~min}$ and then stained with $0.1 \%$ crystal violet for $15 \mathrm{~min}$. The cells on the filters were examined and counted under an inverted microscope. Each experiment was repeated three times.

Xenograft tumor model in nude mice. A total of $5 \mathrm{x}$ $10^{5}$ human gastric cancer SGC7901 cells together with $1 \times 10^{5}$ TAMs induced from THP-1 cells (PMA/IL-4/IL-13) with or without LGMN KD were subcutaneously transplanted into the right flank of nude mice ( $\mathrm{n}=6$ in each group, 4 to 6 weeks old, male, purchased from the Department of Experimental Animal Science, Shanghai Jiao Tong University School of Medicine) using a 1-ml syringe. Tumor size was measured using a Vernier caliper every three days. The animals were sacrificed, and the masses were processed, followed by hematoxylin-eosin (H\&E) staining. The animal study was approved by the Institutional Animal Care and Use Committee of Renji Hospital Affiliated to Shanghai Jiao Tong University School of Medicine.

Immunofluorescence and immunohistochemical assays. Immunofluorescence experiments were performed. Briefly, $2 \times 10^{5}$ cells were seeded on coverslips in each well of a 6-well plate. The cells were washed 3 times with PBS before fixation with $4 \%$ paraformaldehyde. The cells were blocked with PBS containing $1 \%$ goat serum for $30 \mathrm{~min}$. Antibodies were incubated at $4{ }^{\circ} \mathrm{C}$ overnight. The cells were washed 6 times with PBS for a total of 3 hours and incubated with secondary antibodies for 1 hour at room temperature. The samples were observed with a Zeiss laser scanning confocal microscope (LSM Meta 510). Single sections are shown. Images were processed (colored and merged) with Zeiss (LSM 510) software. Immunohistochemical assays were performed. An anti-Ki67 antibody (ab8191, Abcam) was used as the primary antibody.

Statistical analysis. Values are expressed as the mean \pm standard error of the mean. One-way analysis of variance with Tukey's test was performed for comparisons of multiple groups. All statistical analyses were performed using SPSS for Windows v. 17.0 (SPSS, Inc.). A two-tailed $p<0.05$ was considered to indicate a statistically significant difference.

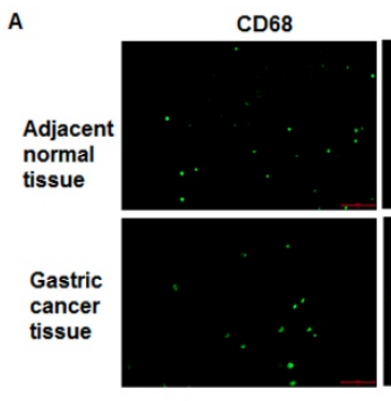

B PMA-Treated THP-1

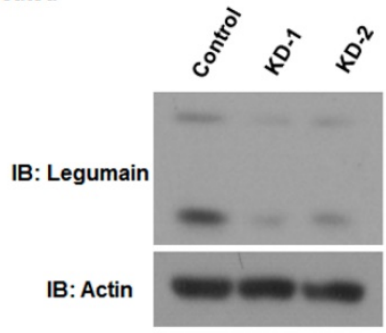
PMA-Treated
THP-1

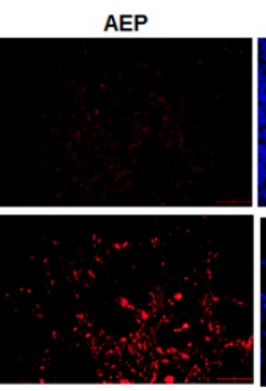

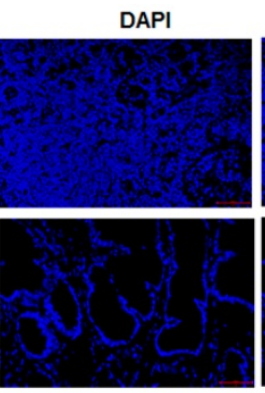

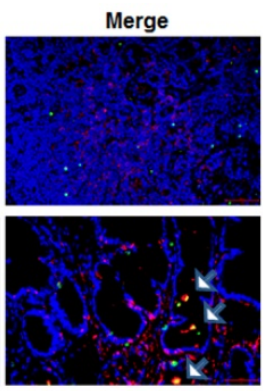

Raw264.7

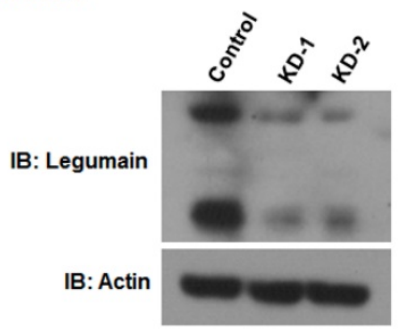

E

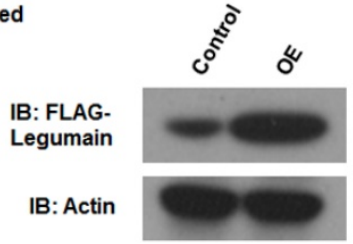

Raw264.7

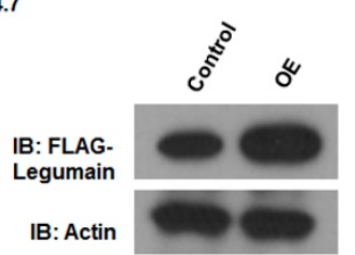

Figure 1. Efficient knockdown or overexpression of LGMN in monocytes/macrophages. (A) Expression of LGMN and CD68 in gastric cancer tissue samples and adjacent normal tissue samples. (B and C) Protein bands for LGMN and $\beta$-actin in THP-1 (PMA/IL-4/IL-13) cells and Raw264.7 (IL-4/IL-13) cells with or without LGMN knockdown. (D and E) Protein bands for FLAG-LGMN and $\beta$-actin in THP-1 (PMA/IL-4/IL-13) cells and Raw264.7 (IL-4/IL-13) cells with or without LGMN overexpression. 


\section{Results}

Efficient knockdown or overexpression of LGMN in TAMs induced from monocytes/macrophages. The expression of legumain in TAMs derived from normal or gastric cancer tissue samples was analyzed. As shown in the revised Figure 1a, legumain was highly expressed in TAMs from the gastric cancer tissue samples compared to those from the adjacent normal tissue samples (Figure 1A). To obtain monocytes/macrophages with stable knockdown or overexpression of LGMN, lentivirus-mediated gene transfection was applied in vitro. A western blot assay was used to detect the efficiency of transfection. The expression of LGMN was significantly knocked down in TAMs induced from THP-1 cells (PMA/IL-4/IL-13) (Figure 1B) and Raw264.7 cells (Figure 1C). LGMN was significantly overexpressed in THP-1 cells (Figure 1D) and Raw264.7 cells (Figure 1E).
Knockdown or overexpression of LGMN in TAMs affected growth and migration. To investigate the function of LGMN in GC, we constructed LGMN-overexpressing (OE) or LGMN-knockdown (KD) THP-1 cell lines and Raw264.7 cell lines. According to growth curve results, we found that LGMN depletion in macrophages significantly reduced the growth of both cell lines compared with that of negative controls (NCs) (Figure $\mathbf{2 A}$ and $\mathbf{B}$ ). In contrast, overexpression of LGMN in macrophages dramatically enhanced cell growth when compared with endogenous expression in control cells (Figure $3 \mathrm{~A}$ and $\mathrm{B}$ ). The results of a transwell migration assay indicated that after LGMN knockdown, the amount of invaded cells was decreased to approximately half that of NC cells in PMA-treated THP-1 cells (Figure 2C) and approximately one third that of NC cells in Raw264.7 cells (Figure 2D). Overexpression of LGMN significantly increased the number of migrating cells in both cell lines (Figure 3C and D).

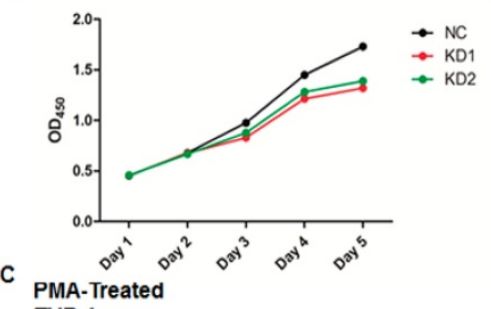

B

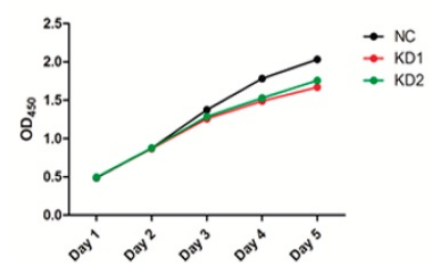

$$
\text { THP-1 }
$$

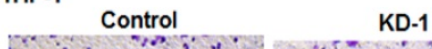

KD-1
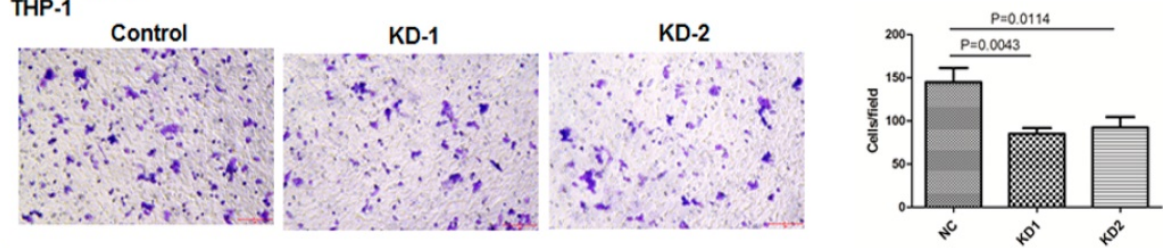

D
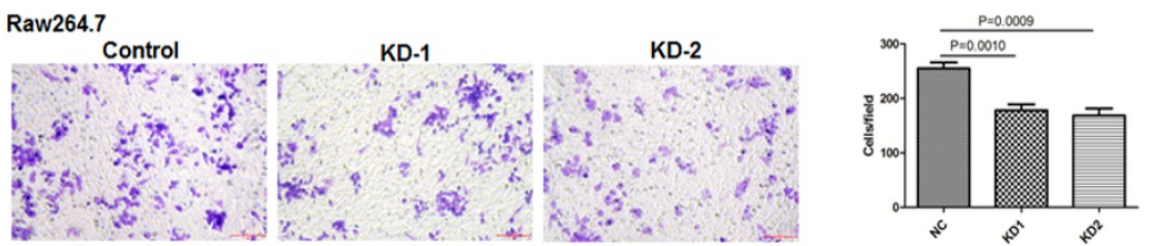

E

MKN28 co-cultured with PMA-treated THP-1

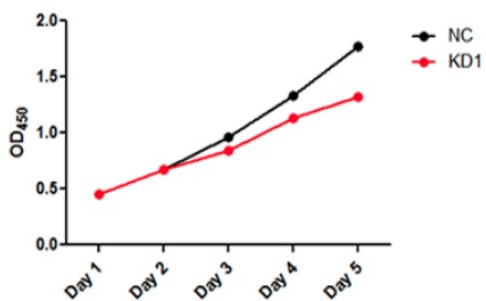

$\mathrm{F}$

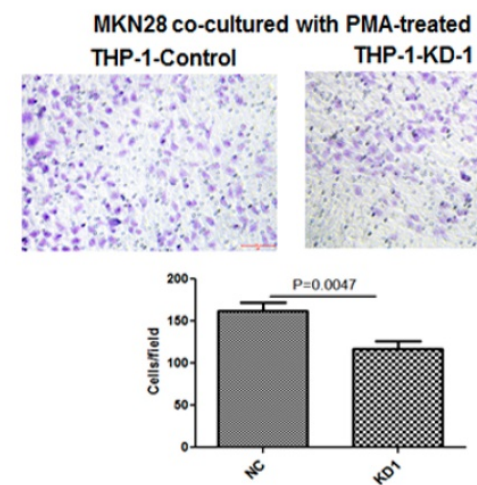

Figure 2. Knocking down LGMN expression in TAMs reduced their activity and migration. (A and B) Growth curves for THP-1 and Raw264.7 cells with or without LGMN knockdown. (C and D) Crystal violet staining after a transwell migration assay and statistical analysis of the cells/field for THP-1 and Raw264.7 cells with or without LGMN knockdown. (E) Growth curves for MKN28 cells cocultured with THP-1 cells with or without LGMN knockdown. (F) Transwell migration assay of MKN28 cells cocultured with THP-1 cells with or without LGMN knockdown. 


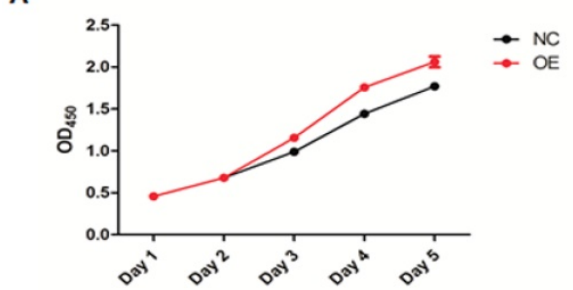

B

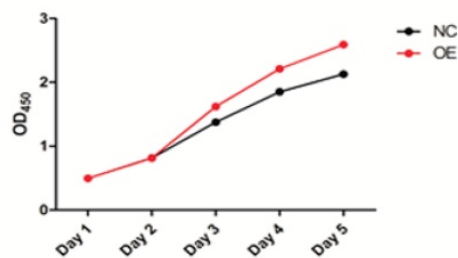

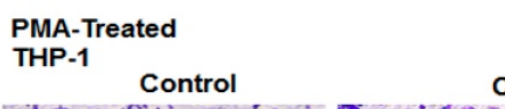

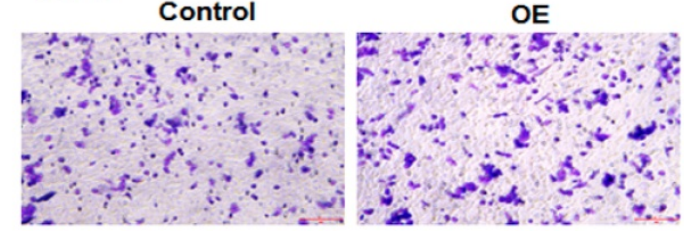

D
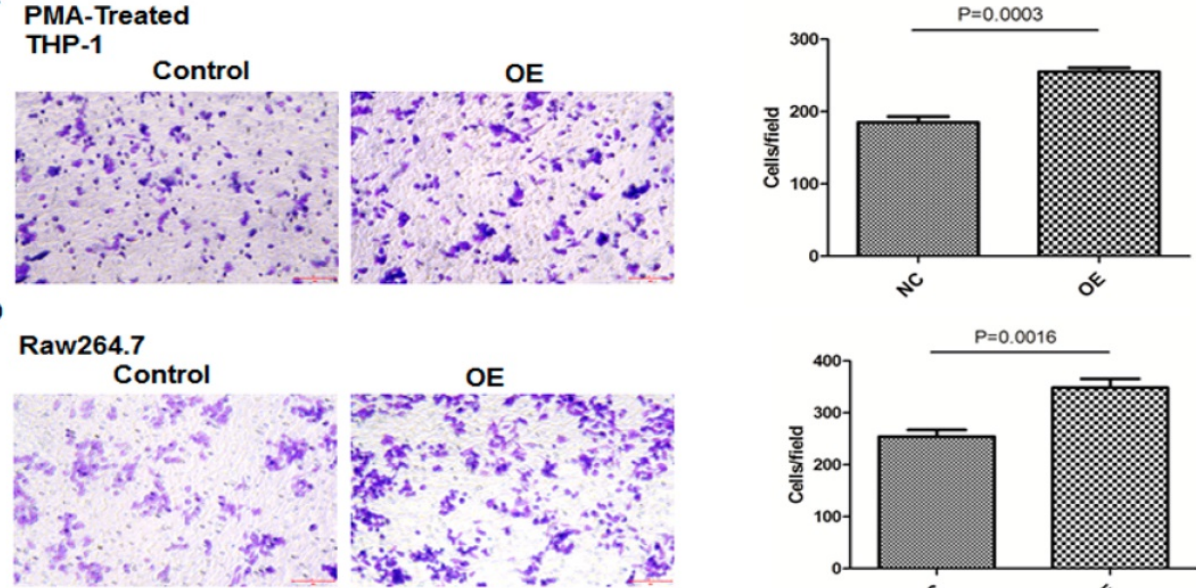

E

\section{$F$}

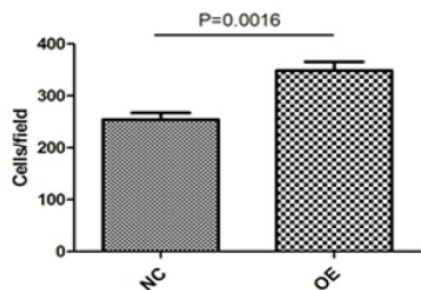

MKN28 co-cultured with PMA-treated THP-1

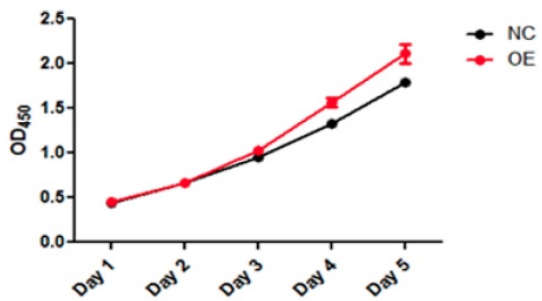

F MKN28 co-cultured with PMA-treated
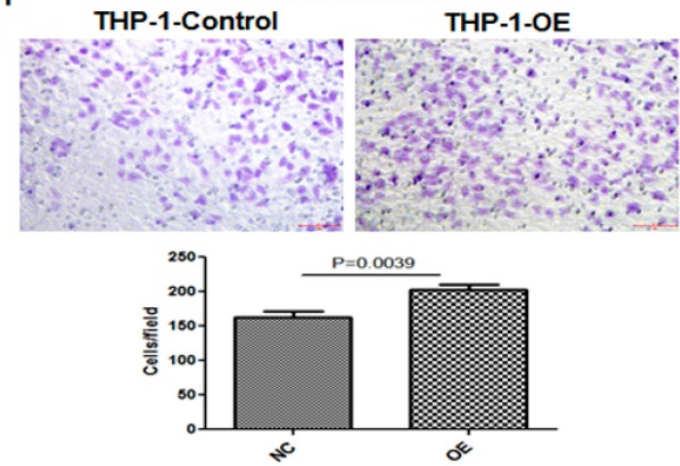

Figure 3. Overexpression of LGMN in TAMs enhanced their activity and migration. (A and B) Growth curves for THP-1 and Raw264.7 cells with or without LGMN knockdown. (C and D) Crystal violet staining after a transwell assay and statistical analysis of the cells/field for THP-1 and Raw264.7 cells with or without LGMN overexpression. (E) Growth curves for MKN28 cells cocultured with THP-1 cells with or without LGMN overexpression. (F) Transwell migration assay of MKN28 cells cocultured with THP-1 cells with or without LGMN overexpression.

CCK-8 and transwell migration assays studying GC cancer cells cocultured with TAMs with or without legumain expression were performed. The results showed that the GC cancer cells cocultured with the legumain-knockdown TAMs showed significantly reduced cellular proliferation and migration (Figure 2E and 2F). However, the MKN28 GC cancer cells cocultured with the legumain-overexpressing TAMs exhibited significantly increased cellular proliferation and migration (Figure 3E and 3F).

Legumain-suppressed TAMs inhibited tumor progression in vivo. For further study of the function of LGMN in TAMs in vivo, LGMN-suppressed Raw264.7 cells were mixed with GC cells and transplanted into nude mice, and the model was constructed successfully. As shown in Figure 4A-C, the size and weight of the tumors in the LGMN-KD group were significantly smaller than those of the tumors in the NC group. H\&E staining revealed many more tumor cells and darker stained nuclei in the NC group than in the LGMN-KD group (Figure 4D).

Legumain-suppressed TAMs reduced tumor growth and angiogenesis in vivo. Results for immunofluorescence staining showed lower expression of APE in LGMN-suppressed TAM-associated tumors than in NC tumors (Figure 5A). Expression of the angiogenesis marker CD31 was clearly suppressed in the LGMN-suppressed TAM-associated tumors (Figure 5B). Immunohistochemistry was used to detect Ki67, a biomarker of malignant growth, and the results indicated downregulation of Ki67 expression in the 
LGMN-suppressed TAM-associated tumors (Figure 5C).

In summary, LGMN played an important role in TAMs. On the one hand, in vitro experiments suggested that altering the expression of LGMN

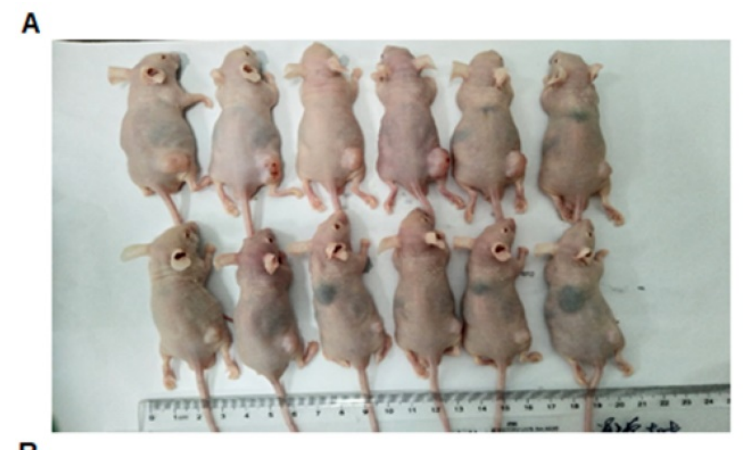

B

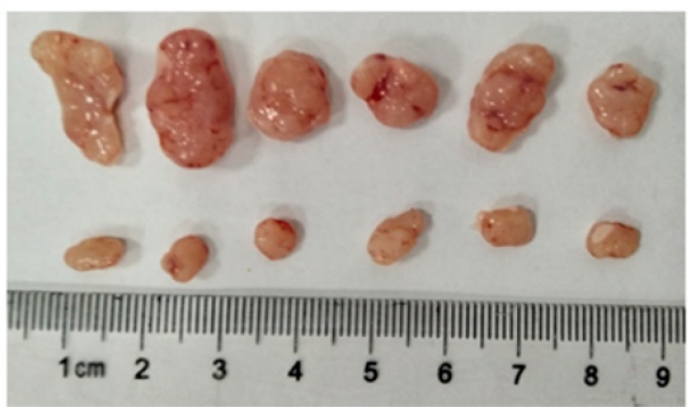

changed the growth and migration of TAMs. On the other hand, in vivo experiments proved that LGMN-suppressed TAMs reduced tumor growth and angiogenesis (Figure 6).

C

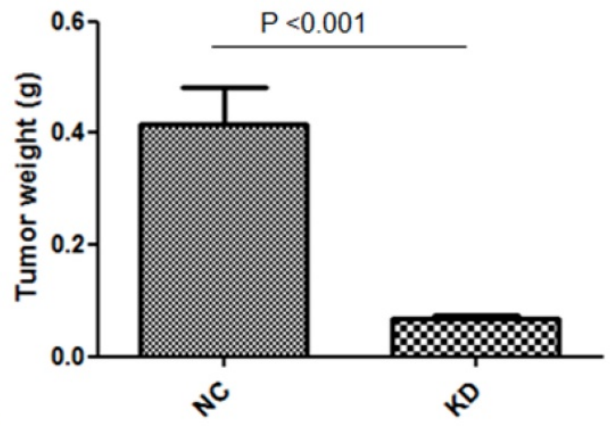

$H \& E$

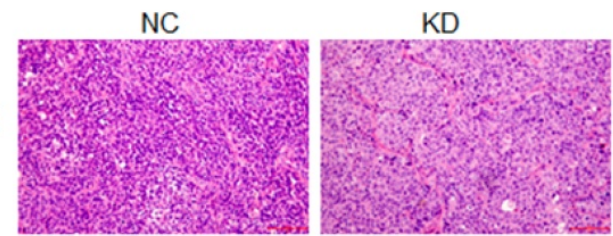

Figure 4. LGMN-KD TAMs reduced tumor development in vivo. (A) Tumor formation after subcutaneous injection of SGC7901 gastric cancer cells mixed with TAMs induced from THP-1 cells (PMA/IL-4/IL-13) with or without LGMN KD. (B) Dissected tumors. (C) Tumor weights for the NC and KD groups. (D) H\&E staining for the NC and KD groups.

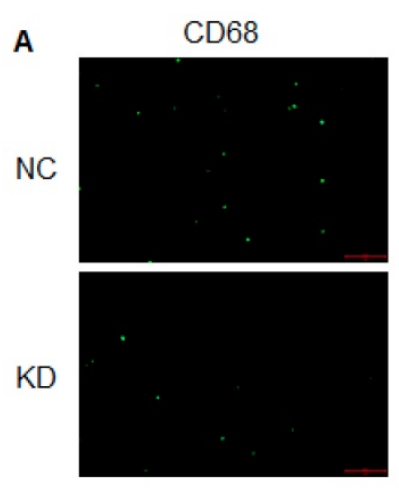

B

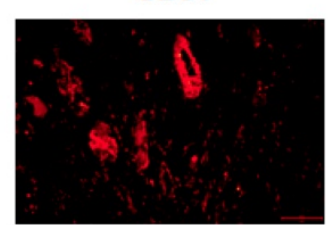

KD

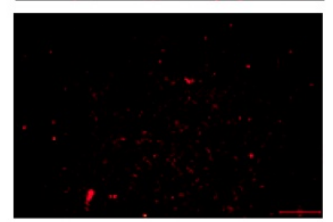

LGMN
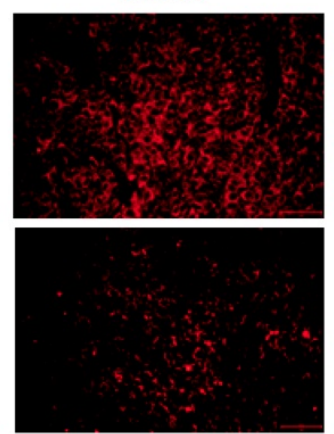

$\mathrm{DAPl}$
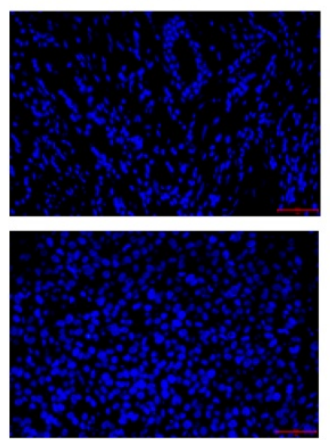

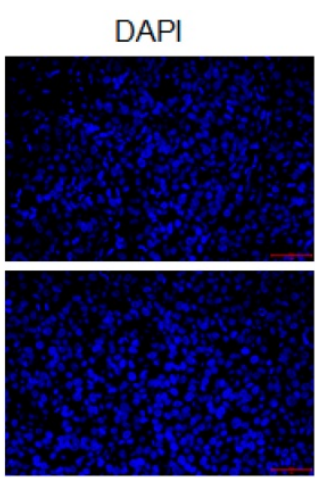

Merge
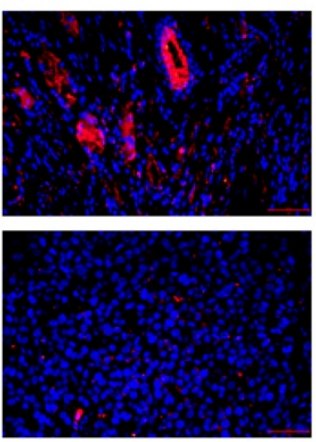

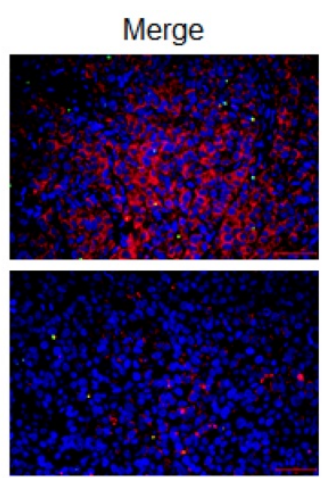

C

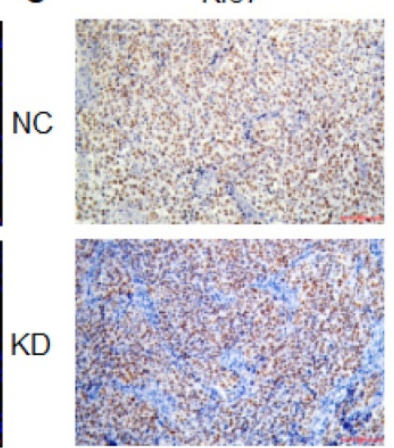

Figure 5. LGMN-KD TAMs reduced tumor cell proliferation and angiogenesis in vivo. (A) Green fluorescence-labeled CD68, red fluorescence-labeled AEP and DAPI-labeled nuclei in LGMN-NC and LGMN-KD tissue samples. (B) Red fluorescence-labeled AEP, DAPI-labeled nuclei and merged images of LGMN-NC and LGMN-KD tissue samples. (C) Immunohistochemical staining for Ki67 in LGMN-NC and LGMN-KD tissue samples. 


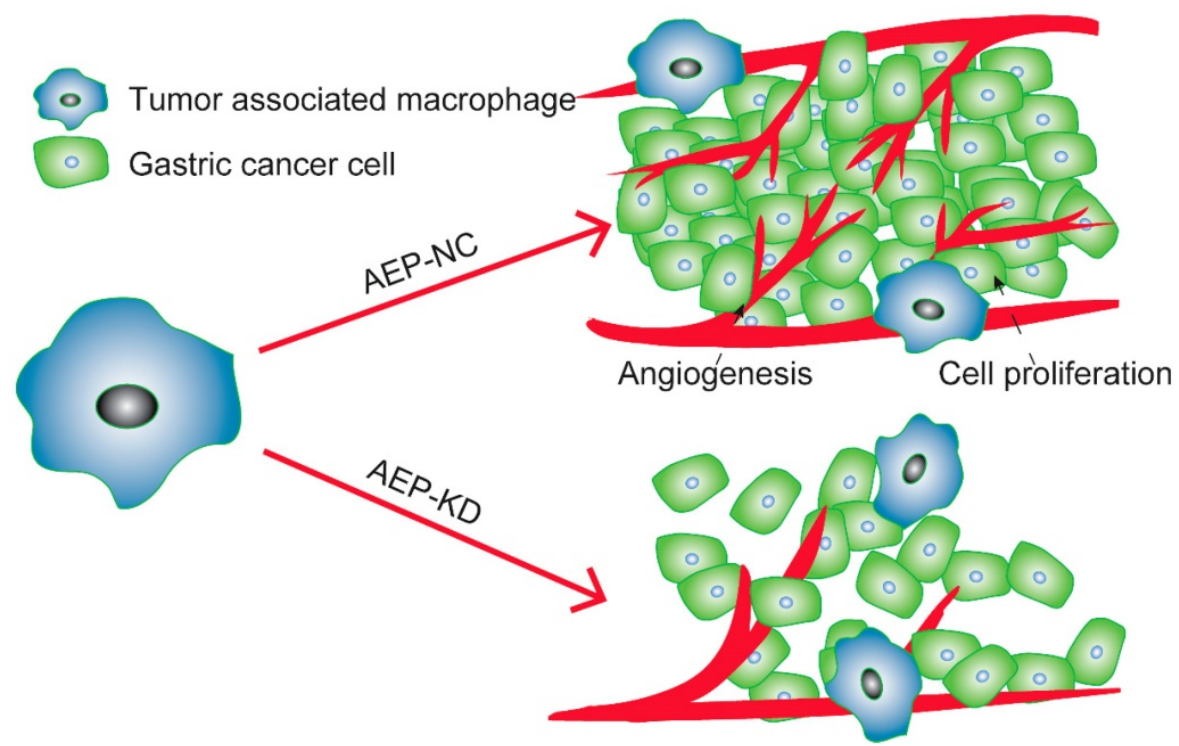

Figure 6. LGMN-KD TAMs reduced tumor cell proliferation and angiogenesis. Diagram of TAMs promoting GC proliferation and angiogenesis.

\section{Discussion}

The tumor microenvironment is a multifarious niche of cells that evolves with and provides support to tumor cells [19-21]. Among the innate and adaptive immune cells recruited to a tumor site, macrophages are particularly abundant and are present at all stages of tumor progression [12, 22]. Tumor infiltration by tumor-associated macrophages is one of the predictive factors in gastric cancers correlated with an unfavorable prognosis [23]. The mechanisms by which macrophages promote tumors are complex. TAMs may promote angiogenesis and lymphangiogenesis in GC, and these effects may be achieved by enhancing VEGF expression [24]. Transfer of TAM-derived miR-21 through exosomes confers cisplatin resistance to gastric cancer cells by activating the PI3K/AKT signaling pathway and downregulating PTEN expression [25]. In addition, TAMs promote the epigenetic silencing of gelsolin through DNA methyltransferase 1 activity induced by CCL5/CCR5/STAT3 signaling in gastric cancer cells [26]. Previous studies found that CXCL8 was a vital driver of GC and it was secreted by macrophages. In turn, CXCL8 inhibited CD8 ${ }^{+} \mathrm{T}$ cell functions by inducing the expression of PD-L1 on macrophages, which eventually promoted tumor progression [27]. TAMs can exert dual influences on cytoreductive therapies, either antagonizing antitumor activity by orchestrating tumor-promoting activity and the tissue-repair response or enhancing the overall antineoplastic effect directly [28, 29]. Currently, immunotherapy shows great potential in cancer treatment; TAMs can express molecular triggers of checkpoint proteins that regulate $\mathrm{T}$ cell activation, which are targets of certain checkpoint blockade immunotherapies [30, 31]. Other macrophageassociated anticancer therapies are under investigation, such as inhibiting macrophage recruitment and survival in tumors; macrophage re-education to induce an antitumor M1 phenotype; and monoclonal antibodies that elicit macrophage-mediated extracellular killing or phagocytosis and intracellular destruction of cancer cells $[11,12,32]$.

LGMN has been proven to be associated with the progression of tumors, and TAMs also play critical roles in promoting angiogenesis, tumor invasion and metastasis. A previous study indicated that tumorpromoting functions may be associated with the expression of LGMN [33-35]. Shen L [36] found that the number of M2 TAMs was negatively correlated with the prognosis of diffuse large B cell lymphoma and that overexpression of LGMN in Raw 264.7 cells promoted the formation of stromal vascular endothelium and degradation of fibronectin and collagen I, which together facilitate tumor progression. Moreover, a series of LGMN substrates including MMPs and p53, which are crucial for tumor progression, have been found [37, 38]. To further understand the roles of LGMN activity in cancer progression and inflammation, Edgington LE [39] designed an activity-based probe that bound active LGMN to trace changes in LGMN in macrophages; the result indicated that the expression of LGMN was highly correlated with macrophage activation and might be an ideal marker for early metastasis. Our study regulated the expression of LGMN in macrophages, which induced significant changes in the growth and metastasis of GC cells. In addition, in 
vivo experiments suggested that the growth and angiogenesis of gastric cancer tumors were suppressed by knocking down the expression of LGMN in TAMs. Together, these results supported and coincided with the findings of reported studies, indicating that LGMN acts as a very important driving factor in TAMs to promote GC progression.

\section{Conclusion}

In conclusion, the current study suggests that LGMN in TAMs performs a novel oncogenic role in the regulation of gastric tumor cell proliferation and invasion in vitro and in vivo. Our findings provide new insights into the molecular details of GC-associated macrophages and propose potential therapeutic targets for this disease.

\section{Abbreviations}

TAMs: tumor-associated macrophages; LGMN: legumin; AEP: asparagine endopeptidase; GC: gastric cancer; NC: negative control; OE: overexpression; TBST: Tris-Buffered Saline and Tween 20; CCK-8: A Cell Counting Kit-8 assay.

\section{Acknowledgment}

The research was funded by Pudong New Area Science and Technology Development Fund Minsheng Research Special Fund Medical and Health Project (PKJ2017-Y30).

\section{Competing Interests}

The authors have declared that no competing interest exists.

\section{References}

1. Siegel RL, Miller KD, Jemal A. Cancer Statistics, 2017. CA: a cancer journal for clinicians. 2017; 67: 7-30.

2. Fitzmaurice C, Dicker D, Pain A, Hamavid H, Moradi-Lakeh M, MacIntyre MF, et al. The Global Burden of Cancer 2013. JAMA oncology. 2015; 1: 505-27.

3. Salomon JA, Wang H, Freeman MK, Vos T, Flaxman AD, Lopez AD, et al. Healthy life expectancy for 187 countries, 1990-2010: a systematic analysis for the Global Burden Disease Study 2010. Lancet (London, England). 2012; 380: 2144-62.

4. Hartgrink HH, Jansen EP, van Grieken NC, van de Velde CJ. Gastric cancer. Lancet (London, England). 2009; 374: 477-90.

5. Zhang X, Li M, Chen S, Hu J, Guo Q, Liu R, et al. Endoscopic Screening in Asian Countries Is Associated With Reduced Gastric Cancer Mortality: A Meta-analysis and Systematic Review. Gastroenterology. 2018; 155: 347-54.e9.

6. Leung WK, Wu MS, Kakugawa Y, Kim JJ, Yeoh KG, Goh KL, et al. Screening for gastric cancer in Asia: current evidence and practice. The Lancet Oncology. 2008; 9: 279-87.

7. Takahari D. Second-line chemotherapy for patients with advanced gastric cancer. Gastric cancer : official journal of the International Gastric Cancer Association and the Japanese Gastric Cancer Association. 2017; 20: 395-406.

8. Wagner AD, Syn NL, Moehler M, Grothe W, Yong WP, Tai BC, et al. Chemotherapy for advanced gastric cancer. The Cochrane database of systematic reviews. 2017; 8: Cd004064.

9. Kim J, Bae JS. Tumor-Associated Macrophages and Neutrophils in Tumor Microenvironment. Mediators of inflammation. 2016; 2016: 6058147.
10. Ngabire D, Kim GD. Autophagy and Inflammatory Response in the Tumor Microenvironment. International journal of molecular sciences. 2017; 18.

11. Mantovani A, Marchesi F, Malesci A, Laghi L, Allavena P. Tumour-associated macrophages as treatment targets in oncology. Nature reviews Clinical oncology. 2017; 14: 399-416.

12. Noy R, Pollard JW. Tumor-associated macrophages: from mechanisms to therapy. Immunity. 2014; 41: 49-61.

13. Ruffell B, Affara NI, Coussens LM. Differential macrophage programming in the tumor microenvironment. Trends in Immunology. 2012; 33: 119-26.

14. Zhen Y, Chunlei G, Wenzhi S, Shuangtao Z, Na L, Rongrong W, et al. Clinicopathologic significance of legumain overexpression in cancer: a systematic review and meta-analysis. Scientific reports. 2015; 5: 16599.

15. Dall E, Brandstetter H. Structure and function of legumain in health and disease. Biochimie. 2016; 122: 126-50.

16. Qi Q, Obianyo O, Du Y, Fu H, Li S, Ye K. Blockade of Asparagine Endopeptidase Inhibits Cancer Metastasis. Journal of medicinal chemistry. 2017; 60: 7244-55.

17. Li N, Liu Q, Su Q, Wei C, Lan B, Wang J, et al. Effects of legumain as a potential prognostic factor on gastric cancers. Medical oncology (Northwood, London, England). 2013; 30: 621.

18. Guo $P$, Zhu Z, Sun Z, Wang Z, Zheng X, Xu H. Expression of legumain correlates with prognosis and metastasis in gastric carcinoma. PloS one. 2013; 8: e73090.

19. De Palma M, Biziato D, Petrova TV. Microenvironmental regulation of tumour angiogenesis. Nature reviews Cancer. 2017; 17: 457-74.

20. Cole SW, Nagaraja AS, Lutgendorf SK, Green PA, Sood AK. Sympathetic nervous system regulation of the tumour microenvironment. Nature reviews Cancer. 2015; 15: 563-72.

21. Condeelis J, Pollard JW. Macrophages: obligate partners for tumor cell migration, invasion, and metastasis. Cell. 2006; 124: 263-6.

22. Dehne N, Mora J, Namgaladze D, Weigert A, Brune B. Cancer cell and macrophage cross-talk in the tumor microenvironment. Current opinion in pharmacology. 2017; 35: 12-9.

23. Chang WJ, Du Y, Zhao X, Ma LY, Cao GW. Inflammation-related factors predicting prognosis of gastric cancer. World journal of gastroenterology. 2014; 20: 4586-96.

24. Wu H, Xu JB, He YL, Peng JJ, Zhang $\mathrm{XH}$, Chen $\mathrm{CQ}$, et al. Tumor-associated macrophages promote angiogenesis and lymphangiogenesis of gastric cancer. Journal of surgical oncology. 2012; 106: 462-8.

25. Zheng P, Chen L, Yuan X, Luo Q, Liu Y, Xie G, et al. Exosomal transfer of tumor-associated macrophage-derived miR-21 confers cisplatin resistance in gastric cancer cells. Journal of experimental \& clinical cancer research : CR. 2017; 36: 53.

26. Wang H-C, Chen C-W, Yang C-L, Tsai IM, Hou Y-C, Chen C-J, et al. Tumor-Associated Macrophages Promote Epigenetic Silencing of Gelsolin through DNA Methyltransferase 1 in Gastric Cancer Cells. Cancer Immunology Research. 2017; 5: 885-97.

27. Lin $\mathrm{C}, \mathrm{He} \mathrm{H}$, Liu H, Li R, Chen $\mathrm{Y}$, Qi Y, et al. Tumour-associated macrophages-derived CXCL8 determines immune evasion through autonomous PD-L1 expression in gastric cancer. Gut. 2019.

28. Deng C, Zhang Q, Jia M, Zhao J, Sun X, Gong T, et al. Tumors and Their Microenvironment Dual-Targeting Chemotherapy with Local Immune Adjuvant Therapy for Effective Antitumor Immunity against Breast Cancer. Advanced science (Weinheim, Baden-Wurttemberg, Germany). 2019; 6: 1801868.

29. Di Caro G, Cortese N, Castino GF, Grizzi F, Gavazzi F, Ridolfi C, et al. Dual prognostic significance of tumour-associated macrophages in human pancreatic adenocarcinoma treated or untreated with chemotherapy. Gut. 2016; 65: 1710-20.

30. Cassetta L, Kitamura T. Macrophage targeting: opening new possibilities for cancer immunotherapy. Immunology. 2018; 155: 285-93.

31. Saha D, Martuza RL, Rabkin SD. Macrophage Polarization Contributes to Glioblastoma Eradication by Combination Immunovirotherapy and Immune Checkpoint Blockade. Cancer cell. 2017; 32: 253-67.e5.

32. Mantovani A, Allavena P. The interaction of anticancer therapies with tumor-associated macrophages. The Journal of experimental medicine. 2015; 212: 435-45

33. Edgington-Mitchell LE, Wartmann T, Fleming AK, Gocheva V, van der Linden WA, Withana NP, et al. Legumain is activated in macrophages during pancreatitis. American journal of physiology Gastrointestinal and liver physiology. 2016; 311: G548-60.

34. Solberg R, Smith R, Almlof M, Tewolde E, Nilsen H, Johansen HT. Legumain expression, activity and secretion are increased during monocyte-to-macrophage differentiation and inhibited by atorvastatin. Biological chemistry. 2015; 396: 71-80. 
35. Wang D, Xiong M, Chen C, Du L, Liu Z, Shi $Y$, et al. Legumain, an asparaginyl endopeptidase, mediates the effect of M2 macrophages on attenuating renal interstitial fibrosis in obstructive nephropathy. Kidney international. 2018; 94: 91-101.

36. Shen L, Li H, Shi Y, Wang D, Gong J, Xun J, et al. M2 tumour-associated macrophages contribute to tumour progression via legumain remodelling the extracellular matrix in diffuse large B cell lymphoma. Scientific reports. 2016; 6: 30347.

37. Bai P, Lyu L, Yu T, Zuo C, Fu J, He Y, et al. Macrophage-Derived Legumain Promotes Pulmonary Hypertension by Activating the MMP (Matrix Metalloproteinase)-2/TGF (Transforming Growth Factor)-beta1 Signaling. Arteriosclerosis, thrombosis, and vascular biology. 2019; 39: e130-e45.

38. Lin Y, Liao K, Miao Y, Qian Z, Fang Z, Yang X, et al. Role of asparagine endopeptidase in mediating wild-type p53 inactivation of glioblastoma. Journal of the National Cancer Institute. 2019.

39. Edgington LE, Verdoes M, Ortega A, Withana NP, Lee J, Syed S, et al. Functional imaging of legumain in cancer using a new quenched activity-based probe. Journal of the American Chemical Society. 2013; 135: $174-82$. 